\title{
THE TENSOR PRODUCT OF SEMIGROUPS
}

\author{
BY \\ PIERRE ANTOINE GRILLET
}

The tensor product of semigroups is defined like the tensor product of modules, by means of multilinear mappings. Surprisingly enough, it has most of the important properties of its homological cousin, together with some others of its own, so that, in view of such results as [4], it is not unreasonable to hope that it will become a very helpful tool for the study of semigroups. The only thing it lacks as an operation is associativity; this is apparently due to the use of noncommutative semigroups throughout this paper, and results in our concentrating on the tensor product of two semigroups, even though such restriction is not necessary for some of our results.

In $\S 1$ we give the definition and some examples: If $B$ is a one-element semigroup, then $A \otimes B$ is the largest idempotent homomorphic image of $A$; if $B$ is an infinite cyclic semigroup, then $A \otimes B$ is the largest normal homomorphic image $N(A)$ of $A$ (normal means that $(x y)^{n}=x^{n} y^{n}$ holds identically for all $n$ ). In $\$ 2$ we prove the existence of the tensor product of any family of semigroups. $\$ 3$ brings a fundamental result, which describes the congruence induced by $f \otimes g$ when the homomorphisms $f$ and $g$ are onto (in which case $f \otimes g$ is also onto). As a first consequence, we prove also that $A \otimes B$ depends only on the largest normal homomorphic images of $A$ and $B$; namely, $A \otimes B$ is naturally isomorphic to $N(A) \otimes N(B)$. In $\S 4$, we prove a very peculiar property of the tensor product of semigroups, namely that it preserves consistent monomorphisms (a semigroup homomorphism is consistent if the complement of its image is an ideal or is empty). The fundamental result of $\$ 3$ can then be extended to consistent morphisms. In $\$ 5$ we show that the tensor product of semigroups is cokernel preserving in the following sense. Call a sequence $A^{\prime} \stackrel{f}{\rightarrow} A \stackrel{f^{\prime}}{\rightarrow} A^{\prime \prime}$ coexact if the congruence induced by $f^{\prime}$ is the smallest in a class in which the image of $f$ is contained. Then the tensor product by any $s$-indecomposable semigroup $X$ preserves such coexact sequences where $f^{\prime}$ is onto (or consistent). We also establish the adjoint associativity. In the right exactness result of $\S 5$, the assumption on $X$ cannot be lifted, but we show in $\S 6$ that this can be fixed by defining another tensor product for semigroups with zero, which is closely related to the tensor product of semigroups and otherwise keeps most of its properties.

Our paper is self-contained except that we do not bother to redefine elementary concepts of semigroup theory, which is better done in [1]. Also, the reader will have

Received by the editors November 18, 1966 and, in revised form, October 23, 1967 and April 1, 1968. 
a better appreciation with a little knowledge of homological algebra (see [6]). We shall denote by $\mathscr{S}$ the category of semigroups; by $\operatorname{Im} f$ the image $f(X)$ of a mapping $f$ of $X$ into $Y(\operatorname{Im} f$ may mean a semigroup if $f$ is a semigroup homomorphism); by $\operatorname{ker} f$, the equivalence relation or congruence induced by $f$.

1. Definition. Examples. If $\left(A_{i}\right)_{i \in I}$ is a family of semigroups, we say that a mapping $s$ of the cartesian product $\prod_{i \in I} A_{i}$ into a semigroup $C$ is I-linear if for every $i_{0} \in I$, the mapping $a_{i_{0}} \leadsto s\left(\left(a_{i}\right)_{i \in I}\right)$ is a homomorphism of $A_{i_{0}}$ into $C$ whenever all $a_{i}$ are fixed except for $a_{i_{0}}$. If an $I$-linear mapping $t$ of $\prod_{i \in I} A_{i}$ into a semigroup $T$ has the property that, for any $I$-linear mapping $s$ of $\prod_{i \in I} A_{i}$ into some semigroup $C$, there exists a unique homomorphism $u$ of $T$ into $C$ such that $s=u \circ t$, then we call the pair $(t, T)$, and also the semigroup $T$, a tensor product of the family $\left(A_{i}\right)_{i \in I}$.

If $\left(A_{i}\right)_{i \in I}$ has a tensor product $(t, T)$ we shall denote $T$ by $\bigotimes_{i \in I} A_{i}$ and $t\left(\left(a_{i}\right)_{i \in I}\right)$ by $\bigotimes_{i \in I} a_{i}$. We shall be mostly concerned with the case when $I$ is finite, $I=\{1,2, \ldots, n\}$; in that case, $\bigotimes_{i \in I} A_{i}$ is also denoted by $A_{1} \otimes A_{2} \otimes \cdots \otimes A_{n}$ and $\bigotimes_{i \in I} a_{i}$ by $a_{1} \otimes a_{2}$ $\otimes \cdots \otimes a_{n}$; I-linear mappings are called also $n$-linear (bilinear, trilinear if $n=2,3$ ).

Before proving that any family of semigroups has a tensor product, we give some examples.

Proposition 1.1. Let $A$ be any semigroup and $E=\{e\}$ be a one-element semigroup. Then any largest idempotent homomorphic image $E(A)$ of $A$ is a tensor product of $A$ and $E$.

Proof. $E(A)$ comes with a homomorphism $f$ of $A$ onto $E(A)$. Then $t:(a, e) \leadsto f(a)$ is a bilinear mapping of $A \times E$ into $E(A)$. Let $s$ be any bilinear mapping of $A \times E$ into some semigroup $D$; then $g: a \leadsto s(a, e)$ is a homomorphism of $A$ into $D$. Furthermore, the image $\operatorname{Im} g$ of $g$ is an idempotent subsemigroup of $D$, for $s(a, e) s(a, e)$ $=s(a, e e)=s(a, e)$ for all $a \in A$. Therefore, there exists a unique homomorphism $u$ of $E(A)$ into $D$ such that $g=u \circ f$; i.e. $s=u \circ t$. This completes the proof.

Call a semigroup $S$ normal if $(x y)^{n}=x^{n} y^{n}$ holds identically in $S$ (cf. [5]). It is clear that any semigroup $A$ has a largest normal homomorphic image; it can be described as quotient of $A$ by the smallest congruence on $A$ containing all pairs of the form $\left((x y)^{n}, x^{n} y^{n}\right)$; we shall denote it by $N(A)$.

Proposition 1.2. Let $A$ be any semigroup and $B$ be an infinite cyclic semigroup generated by $b$. Then $N(A)$ is a tensor product of $A$ and $B$.

Proof. Let $f$ be the canonical homomorphism of $A$ onto $N(A)$. Since $N(A)$ is normal, $t:\left(a, b^{n}\right) m f\left(a^{n}\right)$ is a bilinear mapping of $A \times B$ into $N(A)$. Let $s$ be any bilinear mapping of $A \times B$ into some semigroup $D$. Then $g: a m s(a, b)$ is a homomorphism of $A$ into $D$. Observing that $s\left(x, y^{n}\right)=s(x, y)^{n}=s\left(x^{n}, y\right)$ holds identically for any bilinear mapping $s$, we conclude that

$$
\begin{aligned}
s\left((x y)^{n}, b\right) & =s\left(x y, b^{n}\right)=s\left(x, b^{n}\right) s\left(y, b^{n}\right) \\
& =s\left(x^{n}, b\right) s\left(y^{n}, b\right)=s\left(x^{n} y^{n}, b\right),
\end{aligned}
$$


for all $x, y \in A$ and all $n$. Therefore $\operatorname{Im} g$ is a normal subsemigroup of $D$. Hence there is a unique homomorphism $u$ of $N(A)$ into $D$ such that $g=u \circ f$; since $s\left(a, b^{n}\right)$ $=s\left(a^{n}, b\right)=g\left(a^{n}\right)=u\left(f\left(a^{n}\right)\right)=u\left(t\left(a, b^{n}\right)\right)$ identically, we have also $s=u \circ t$. Conversely, if $v$ is any homomorphism such that $s=v \circ t$, then also $g=v \circ f$ so that $v=u$ is unique. This completes the proof.

Using a similar reasoning, one can show easily that:

Proposition 1.3. Let $A$ be any semigroup and $B$ be a cyclic semigroup generated by an element $b$, subject to the relation $b^{n}=b^{n+P}$ (where $n, P>0$ ). Let $C$ be a largest homomorphic image of $A$ such that $C$ is normal and the identity $x^{n}=x^{n+P}$ holds in $C$. Then $C$ is a tensor product of $A$ and $B$.

From this it is easily deduced that the tensor product of a cyclic semigroup of index $n$ and period $p$ and a cyclic semigroup of index $m$ and period $q$ is again a cyclic semigroup of index inf $(n, m)$ and of period the g.c.d. of $p$ and $q$. In particular, the tensor product of two finite cyclic groups is a finite cyclic group. More generally,

Proposition 1.4. If $A$ and $B$ are groups, then $A \otimes B$ is a group.

Proof. We may anticipate and use here the general results of the next section, which insure the existence of $A \otimes B$ and the fact that it is generated by all elements of the form $a \otimes b$. Let $e(f)$ be the identity of $A(B)$. For any $b^{\prime} \in B, e \otimes b^{\prime}$ is an identity element of $A \otimes B$; indeed, if $a \otimes b$ is any generator of $A \otimes B$, and if $b=b^{\prime} u$, then, by bilinearity,

$$
\left(e \otimes b^{\prime}\right)(a \otimes b)=\left(e \otimes b^{\prime}\right)\left(a \otimes b^{\prime}\right)(a \otimes u)=\left(a \otimes b^{\prime}\right)(a \otimes u)=a \otimes b
$$

and, similarly, $(a \otimes b)\left(e \otimes b^{\prime}\right)=a \otimes b$ (writing $\left.b=v b^{\prime}\right)$. Likewise, $a \otimes f$ is an identity element of $A \otimes B$ for all $a \in A$. In particular, $a \otimes f=e \otimes f=e \otimes b$ for all $a, b$.

If now $a^{-1}$ is the inverse of $a$ in $A$, then

$$
\left(a^{-1} \otimes b\right)(a \otimes b)=e \otimes b=(a \otimes b)\left(a^{-1} \otimes b\right),
$$

which shows that any generator of $A \otimes B$ has an inverse. Therefore, $A \otimes B$ is a group.

Since the universal property of $A \otimes B$ applies in particular for bilinear mappings of $A \times B$ into a group, one may restate Proposition 1.4 as follows: The tensor product of two semigroups has a restriction to the variety of groups, which is precisely the tensor product in that variety. (We shall not say more about the tensor product in the variety of all groups, for the following reasons. Observe that a group is normal if and only if it is abelian. Then it will follow from Proposition 3.7 that the tensor product of two groups $A$ and $B$ depends only on the abelian groups $A /[A, A], B /[B, B]$. Thus we do not think that it can be an interesting tool.) 


\section{Existence and first properties.}

THEOREM 2.1. Let $\left(A_{i}\right)_{i \in I}$ be any family of semigroups. There exists a tensor product of that family and it is unique up to isomorphism.

Proof. The uniqueness is clear from the definition of the tensor product as a solution of a universal problem. To show the existence, we construct one as follows. Since this construction will be used again later, we give the notation first.

Notation 2.2. Let $F$ be a free semigroup on the set $\prod_{i \in I} A_{i}$ and $w$ be the canonical inclusion of $\prod_{i \in I} A_{i}$ into $F$. Let $\mathscr{B}$ be the set of all pairs of the form

$$
\left(w\left(\left(a_{i}\right)_{i \in I}\right), w\left(\left(a_{i}^{\prime}\right)_{i \in I}\right) w\left(\left(a_{i}^{\prime \prime}\right)_{i \in I}\right)\right)
$$

where $a_{i}, a_{i}^{\prime}, a_{i}^{\prime \prime} \in A_{i}$ are such that $a_{i_{0}}=a_{i_{0}}^{\prime} a_{i_{0}}^{\prime \prime}$ for some index $i_{0}$, and $a_{i}=a_{i}^{\prime}=a_{i}^{\prime \prime}$ for all other indexes. Let $\mathscr{C}$ be the smallest congruence on $F$ containing $\mathscr{B}$. Let $T=F / \mathscr{C}$, $k$ be the canonical homomorphism of $F$ onto $T$ and $t=k \circ w$.

This construction provides us with a pair $(t, T)$ which is a tensor product of the given family. Indeed $t$ is an I-linear mapping by the choice of $\mathscr{C}$. Let $s$ be any $I$-linear mapping of $\prod_{i \in I} A_{i}$ into some semigroup $C$. First there exists a unique homomorphism $f$ of $F$ into $C$ such that $s=f \circ w$. Since $s$ is $I$-linear, ker $f$ contains all pairs of $\mathscr{C}$; therefore $\operatorname{ker} k=\mathscr{C} \subseteq \operatorname{ker} f$, so that $f=u \circ k$ for some unique homomorphism $u$ of $T$ into $C$. We see that $u$ is such that $s=f \circ w=u \circ k \circ w=u \circ t$. Finally any homomorphism $v$ of $T$ into $D$ such that $s=v \circ t(=v \circ k \circ w)$ is such that $v \circ k=f$ by uniqueness of $f$, so that $v=u$ is unique. This completes the proof.

Using the same method, it can be proved that any variety has a tensor product of its own.

The construction above provides us with a presentation of the tensor product. For example, if we start with two semigroups $A, B$, we may construct $A \otimes B$ as the semigroup generated by all "symbols" $a \otimes b$, subject to all the bilinearity relations. Therefore, if $A$ and $B$ are finitely generated, or finitely presented, so is $A \otimes B$. The following example uses this description to show that, if $A$ and $B$ are finite, $A \otimes B$ need not be finite.

EXAMPLE 2.3. Let $A$ and $B$ be semigroups of order 2 consisting of a zero and an identity. Then the tensor product of $A$ and $B$ is the semigroup generated by $0=0 \otimes 0,1=1 \otimes 1, e=0 \otimes 1, f=1 \otimes 0$ subject to all bilinearity relations: $00=0,11=1, e e=e, f f=f, 0 e=e 0=0=0 f=f 0, e 1=1 e=e, f 1=1 f=f$. From these relations it follows that $01=0 e 1=0 e=0$ and, similarly, $10=0$; hence 0 is a zero of $A \otimes B, 1$ is an identity of $A \otimes B$, and we can describe $A \otimes B$ as $C \cup\{0,1\}$, where $C$ is the semigroup generated by $e, f$ subject to the relations $e^{2}=e, f^{2}=f$. It is immediate to verify that any element of $C$ can be written uniquely as either $(e f)^{n}$ or $(f e)^{n}$ or $(e f)^{p} e$ or $(f e)^{p} f$ for some $n>0$ or some $p \geqq 0$. Thus $C$ is infinite. Therefore $A \otimes B$ is also infinite.

It is easy to verify that the tensor product is a commutative operation (e.g. $A \otimes B \cong B \otimes A$ ), but it fails to be associative (we mean, of course, the tensor 
product of semigroups). A counterexample is furnished by Example 2.3. Since $B$ is idempotent there, $B \otimes E \cong B$ by 1.1 , so that $A \otimes(B \otimes E) \cong A \otimes B$. However $(A \otimes B) \otimes E$, which is an idempotent semigroup by Proposition 1.1, is not isomorphic to $A \otimes B$ since ef, for example, is not idempotent. (A description of $(A \otimes B) \otimes E$ is easily obtained from Proposition 1.1 as the quotient of $A \otimes B$ by the congruence whose classes are $\{0\},\{1\},\{e\},\{f\},\left\{(e f)^{n} ; n>0\right\},\left\{(f e)^{n} ; n>0\right\}$, $\left\{(e f)^{n} e ; n>0\right\},\left\{(f e)^{n} f ; n>0\right\}$.) It turns out that in this case $A \otimes B \otimes E \cong A \otimes$ $(B \otimes E)$. All we can say when $A, B, C$ are any semigroups is that there exist canonical homomorphisms of $A \otimes B \otimes C$ onto $(A \otimes B) \otimes C$ and $A \otimes(B \otimes C)$.

Finally, it follows at once from the definition that, if $\left(A_{i}\right)_{i \in I},\left(B_{i}\right)_{i \in I}$ are families of semigroups and if a homomorphism $f_{i}$ of $A_{i}$ into $B_{i}$ is given for each $i \in I$, then there exists a unique homomorphism of $\bigotimes_{i \in I} A_{i}$ into $\bigotimes_{i \in I} B_{i}$, which shall be denoted by $\bigotimes_{i \in I} f_{i}$, such that $\left(\bigotimes_{i \in I} f_{i}\right)\left(\bigotimes_{i \in I} a_{i}\right)=\bigotimes_{i \in I} f_{i}\left(a_{i}\right)$ identically. By Theorem 2.1 it is possible to choose a tensor product for any family and any such choice defines then a functor, covariant in each variable.

3. Action on onto homomorphisms. Throughout this section, $f(g)$ is a given semigroup homomorphism of $A(B)$ into $A^{\prime}\left(B^{\prime}\right)$. To study $f \otimes g$ we shall have to use the tensor product construction of $\$ 2$; in such case, we shall use the following

Notation 3.1. We use the Notation 2.2 for the construction of $A \otimes B$ and $A^{\prime} \otimes B^{\prime}$; primes will refer to $A^{\prime} \otimes B^{\prime}$. In addition, $h$ denotes the mapping of $A \times B$ into $A^{\prime} \times B^{\prime}$ defined by: $h(a, b)=(f(a), g(b)) ; \bar{h}$ denotes the unique homomorphism of $F$ into $F^{\prime}$ such that $\bar{h} \circ w=w^{\prime} \circ h$.

By the definition of $f \otimes g$, we see that $k^{\prime} \circ \bar{h} \circ w=k^{\prime} \circ w^{\prime} \circ h=(f \otimes g) \circ k \circ w$, so that $k^{\prime} \circ \bar{h}=(f \otimes g) \circ k$.

Proposition 3.2. If $f$ and $g$ are onto, then $f \otimes g$ is onto.

Proof. Then $h$ is onto, so that $\bar{h}$ is onto; hence $(f \otimes g) \circ k=k^{\prime} \circ \bar{h}$ is onto and therefore $f \otimes g$ is onto.

Now we want to describe $\operatorname{ker} f \otimes g$.

Definition 3.3. If $\mathscr{K}(\mathscr{L})$ is a congruence on $A(B), \mathscr{K} \otimes \mathscr{L}$ is the smallest congruence on $A \otimes B$ containing all pairs $\left(a_{1} \otimes b_{1}, a_{2} \otimes b_{2}\right)$ such that $\left(a_{1}, a_{2}\right) \in \mathscr{K}$, $\left(b_{1}, b_{2}\right) \in \mathscr{L}$.

THEOREM 3.4. Without any assumption on $f, g: \operatorname{ker} f \otimes \operatorname{ker} g \subseteq \operatorname{ker} f \otimes g$. If $f$ and $g$ are onto, then the equality holds.

Proof. If $\left(a_{1}, a_{2}\right) \in \operatorname{ker} f,\left(b_{1}, b_{2}\right) \in \operatorname{ker} g$, then

$$
(f \otimes g)\left(a_{1} \otimes b_{1}\right)=f\left(a_{1}\right) \otimes g\left(b_{1}\right)=f\left(a_{2}\right) \otimes g\left(b_{2}\right)=(f \otimes g)\left(a_{2} \otimes b_{2}\right)
$$

and $\left(a_{1} \otimes b_{1}, a_{2} \otimes b_{2}\right) \in \operatorname{ker} f \otimes g$. Therefore $\operatorname{ker} f \otimes \operatorname{ker} g \subseteq \operatorname{ker} f \otimes g$.

To prove the converse inclusion in the case when $f$ and $g$ are onto, consider the quotient $C$ of $A \otimes B$ by $\operatorname{ker} f \otimes \operatorname{ker} g$, and the canonical homomorphism $p$ of $A \otimes B$ onto $C$. We show first that $\operatorname{ker} \bar{h} \subseteq \operatorname{ker} p \circ k$. 
Indeed, suppose that $x_{1}, \ldots, x_{n}, y_{1}, \ldots, y_{p} \in A, z_{1}, \ldots, z_{n}, t_{1}, \ldots, t_{p} \in B$ are such that

$$
\bar{h}\left(w\left(x_{1}, z_{1}\right) \cdots w\left(x_{n}, z_{n}\right)\right)=\bar{h}\left(w\left(y_{1}, t_{1}\right) \cdots w\left(y_{p}, t_{p}\right)\right) .
$$

Then, successively,

$$
\begin{gathered}
w^{\prime}\left(f\left(x_{1}\right), g\left(z_{1}\right)\right) \cdots w^{\prime}\left(f\left(x_{n}\right), g\left(z_{n}\right)\right)=w^{\prime}\left(f\left(y_{1}\right), g\left(t_{1}\right)\right) \cdots w^{\prime}\left(f\left(y_{p}\right), g\left(t_{p}\right)\right) ; \\
n=p \quad \text { and } f\left(x_{i}\right)=f\left(y_{i}\right), \quad g\left(z_{i}\right)=g\left(t_{i}\right) \quad \text { for all } i ; \\
\left(k\left(w\left(x_{i}, z_{i}\right)\right), k\left(w\left(y_{i}, t_{i}\right)\right)\right)=\left(x_{i} \otimes z_{i}, y_{i} \otimes t_{i}\right) \in \operatorname{ker} f \otimes \operatorname{ker} g=\operatorname{ker} p \text { for all } i ; \\
p\left(k\left(w\left(x_{1}, z_{1}\right) \cdots w\left(x_{n}, z_{n}\right)\right)\right)=p\left(k\left(w\left(y_{1}, t_{1}\right) \cdots w\left(y_{p}, t_{p}\right)\right)\right) .
\end{gathered}
$$

Therefore $\operatorname{ker} \bar{h} \subseteq \operatorname{ker} p \circ k$.

Since $\bar{h}$ is onto, there is a homomorphism $v$ of $F^{\prime}$ into $C$ such that $p \circ k=v \circ \bar{h}$. Then $v \circ w^{\prime} \circ h=v \circ \bar{h} \circ w=p \circ k \circ w$ is bilinear. Thus $v \circ w^{\prime}$ is also bilinear and can be written under the form $v \circ w^{\prime}=u \circ k^{\prime} \circ w^{\prime}$ for some homomorphism $u$ of $A^{\prime} \otimes B^{\prime}$ into $C$. Finally

$$
p \circ k \circ w=v \circ w^{\prime} \circ h=u \circ k^{\prime} \circ w^{\prime} \circ h=u \circ(f \otimes g) \circ k \circ w,
$$

so that $p=u \circ(f \otimes g)$ and $\operatorname{ker} f \otimes g \subseteq \operatorname{ker} p=\operatorname{ker} f \otimes \operatorname{ker} g$. This completes the proof.

COROllaRY 3.5. If $\mathscr{K}(\mathscr{L})$ is a congruence on $A(B)$, then $A / \mathscr{K} \otimes B / \mathscr{L} \cong$ $A \otimes B / \mathscr{K} \otimes \mathscr{L}$.

The following lemma is useful.

LeMmA 3.6. Suppose that $\mathscr{K}(\mathscr{L})$ is the smallest congruence on $A(B)$ containing a given binary relation $\mathscr{P}(\mathscr{Q})$. Then $\mathscr{K} \otimes \mathscr{L}$ is the smallest congruence on $A \otimes B$ containing all pairs $\left(a^{\prime} \otimes b, a^{\prime \prime} \otimes b\right)$ such that $\left(a^{\prime}, a^{\prime \prime}\right) \in \mathscr{P}$ and all pairs $\left(a \otimes b^{\prime}\right.$, $\left.a \otimes b^{\prime \prime}\right)$ such that $\left(b^{\prime}, b^{\prime \prime}\right) \in \mathscr{Q}$.

Proof. Clearly $\mathscr{K} \otimes \mathscr{L}$ contains all these pairs, so that, if $\mathscr{M}$ is the smallest congruence with that property, $\mathscr{M} \subseteq \mathscr{K} \otimes \mathscr{L}$. To prove the converse inclusion, for every $b \in B$ let $\mathscr{M}_{b}$ be the binary relation on $A$ defined by: $\left(a^{\prime}, a^{\prime \prime}\right) \in \mathscr{M}_{b}$ if and only if $\left(a^{\prime} \otimes b, a^{\prime \prime} \otimes b\right) \in \mathscr{M}$. Since $a \leadsto a \otimes b$ is a homomorphism, $\mathscr{M}_{b}$ is in fact a congruence; also $\mathscr{P} \subseteq \mathscr{M}_{b}$ by definition of $\mathscr{M}$. Therefore $\mathscr{K} \subseteq \mathscr{M}_{b}$; in other words, $\left(a^{\prime}, a^{\prime \prime}\right) \in \mathscr{K}$ implies $\left(a^{\prime} \otimes b, a^{\prime \prime} \otimes b\right) \in \mathscr{M}$. Similarly, $\left(b^{\prime}, b^{\prime \prime}\right) \in \mathscr{L}$ implies $\left(a \otimes b^{\prime}\right.$, $\left.a \otimes b^{\prime \prime}\right) \in \mathscr{M}$. Therefore $\left(a^{\prime}, a^{\prime \prime}\right) \in \mathscr{K}$ and $\left(b^{\prime}, b^{\prime \prime}\right) \in \mathscr{L}$ implies $\left(a^{\prime} \otimes b^{\prime}, a^{\prime \prime} \otimes b^{\prime}\right) \in \mathscr{M}$, $\left(a^{\prime \prime} \otimes b^{\prime}, a^{\prime \prime} \otimes b^{\prime \prime}\right) \in \mathscr{M}$ and eventually $\left(a^{\prime} \otimes b^{\prime}, a^{\prime \prime} \otimes b^{\prime \prime}\right) \in \mathscr{M}$. Thus $\mathscr{K} \otimes \mathscr{L} \subseteq \mathscr{M}$, which completes the proof.

As an application of these results, we show that $A \otimes B$ depends only on $N(A)$ and $N(B)$. More precisely,

Proposition 3.7. For any two semigroups $A$ and $B, A \otimes B \cong N(A) \otimes N(B)$, by an isomorphism which is natural in $A$ and $B$. 
Proof. Let $f(g)$ be the canonical homomorphism of $A(B)$ onto $N(A)(N(B))$. By Proposition 3.2, Theorem 3.4, and Lemma 3.6, $f \otimes g$ is a homomorphism of $A \otimes B$ onto $N(A) \otimes N(B)$ and ker $f \otimes g$ is the smallest congruence on $A \otimes B$ containing all pairs $\left((x y)^{n} \otimes b, x^{n} y^{n} \otimes b\right),\left(a \otimes(z t)^{n}, a \otimes z^{n} t^{n}\right)$, where $a, x, y \in A$, $b, z, t \in B$. But

$$
\begin{aligned}
(x y)^{n} \otimes b & =x y \otimes b^{n}=\left(x \otimes b^{n}\right)\left(y \otimes b^{n}\right) \\
& =\left(x^{n} \otimes b\right)\left(y^{n} \otimes b\right)=x^{n} y^{n} \otimes b
\end{aligned}
$$

and similarly $a \otimes(z t)^{n}=a \otimes z^{n} t^{n}$ identically. Therefore ker $f \otimes g$ is the equality. Then $f \otimes g$ is an isomorphism of $A \otimes B$ onto $N(A) \otimes N(B)$. Naturality is clear.

It is to be observed that a tensor product of two (normal) semigroups is not necessarily normal. For instance, in Example 2.3, $\left(c_{0} d_{0}\right)^{n}=\left(a_{1}\right)^{n}=a_{n},\left(c_{0}\right)^{n}\left(d_{0}\right)^{n}$ $=c_{0} d_{0}=a_{1}$.

4. Action on one-to-one homomorphisms. Coming back to the general situation of $\S 3$, we assume now that $f$ and $g$ are one-to-one. In this case $f \otimes g$ need not be one-to-one, as shown by the following example. Let $A, A^{\prime}, B=B^{\prime}$ be cyclic groups of order 2, 4, 6 generated by $a, a^{\prime}, b$, respectively. By the remark following Proposition 1.3, $A \otimes B, A^{\prime} \otimes B$ are groups of order 2 , generated by $a \otimes b, a^{\prime} \otimes b$, respectively. Let $f$ be defined by $f(a)=a^{\prime 2}$ and $g=\mathrm{id}_{B}$, so that $f$ and $g$ are one-to-one. Then $f \otimes g$ is defined by: $(f \otimes g)(a \otimes b)=a^{\prime 2} \otimes b=\left(a^{\prime} \otimes b\right)^{2}=1$; it is constant hence not one-to-one.

Yet there is one case when we can say that $f \otimes g$ is one-to-one. Recall that a subset $K$ of a semigroup $S$ is consistent (cf. [2]) if $x y \in K$ implies $x \in K$ and $y \in K$; in other words, if $S-K$ is an ideal or is empty. We say that $f, g$ are consistent if $\operatorname{Im} f, \operatorname{Im} g$ are.

THEOREM 4.1. If $f$ and $g$ are one-to-one and consistent, then $f \otimes g$ is one-to-one and consistent.

Proof. The proof uses the tensor product construction of $\$ 2$; we shall keep the notation in 2.2 and 3.1. Furthermore, we shall need the complete description of $\mathscr{C}, \mathscr{C}^{\prime}$ from $\mathscr{B}, \mathscr{B}$, for which we use the following

Notation 4.2. Let $\overline{\mathscr{B}}$ be the binary relation on $F$ defined by: $(M, N) \in \overline{\mathscr{B}}$ if and only if either $(M, N) \in \mathscr{B}$ or $(N, M) \in \mathscr{B}$ or $M=N$. Let $\mathscr{T}$ be the binary relation on $F$ defined by: $(M, N) \in \mathscr{T}$ if and only if $M=U P V, N=U Q V$ for some $U, V \in F^{1}$, $P, Q \in F$ such that $(P, Q) \in \overline{\mathscr{B}}$. Then $\mathscr{C}$ is the transitive closure of $\mathscr{T}$. The (formal) identity of $F^{1}$ can be considered as the "empty word" and $\bar{h}$ shall be extended to $F^{1}$ by $\bar{h}(1)=1$.

Coming back to the proof of the theorem, we first observe that, if we can prove that $(\bar{h}(M), \bar{h}(N)) \in \mathscr{C}^{\prime}$ implies $(M, N) \in \mathscr{C}$, then it will follow that $(f \otimes g)(k(M))$ $=(f \otimes g)(k(N))$ implies $k(M)=k(N)$, i.e. that $f \otimes g$ is one-to-one (since $k$ is onto). This will be established using the construction above and the two following general properties of $\bar{h}$ : (1) $\bar{h}$ preserves the length of words; (2) $\bar{h}$ is consistent; more 
precisely, if $\bar{h}(M)=P^{\prime} Q^{\prime}$, then, since $F^{\prime}$ is free, there exists $P, Q \in F$ such that $P^{\prime}=\bar{h}(P), Q^{\prime}=\bar{h}(Q), M=P Q$. Furthermore, $\bar{h}$ is one-to-one.

If first $(\bar{h}(M), \bar{h}(N)) \in \mathscr{B}^{\prime}$, then, for instance, $\bar{h}(M)=w^{\prime}\left(a_{1}^{\prime} a_{2}^{\prime}, b^{\prime}\right)$,

$$
\bar{h}(N)=w^{\prime}\left(a_{1}^{\prime}, b^{\prime}\right) w^{\prime}\left(a_{2}^{\prime}, b^{\prime}\right)
$$

for some $a_{1}^{\prime}, a_{2}^{\prime} \in A^{\prime}, b^{\prime} \in B^{\prime}$. Now $\bar{h}$ preserves length and so we must have $M$ $=w(a, b), N=w\left(a_{1}, b_{1}\right) w\left(a_{2}, b_{2}\right)$ for some $a, a_{1}, a_{2} \in A, b, b_{1}, b_{2} \in B$, which must satisfy $f(a)=a_{1}^{\prime} a_{2}^{\prime}, f\left(a_{1}\right)=a_{1}^{\prime}, f\left(a_{2}\right)=a_{2}^{\prime}, g(b)=g\left(b_{1}\right)=g\left(b_{2}\right)=b^{\prime}$. Since $f$ and $g$ are one-to-one, we have $a=a_{1} a_{2}, b=b_{1}=b_{2}$ so that $(M, N) \in \mathscr{B}$. The other case, when $\bar{h}(M)=w^{\prime}\left(a^{\prime}, b_{1}^{\prime} b_{2}^{\prime}\right), \bar{h}(N)=w^{\prime}\left(a^{\prime}, b_{1}^{\prime}\right) w^{\prime}\left(a^{\prime}, b_{2}^{\prime}\right)$ is treated similarly.

Furthermore, $\left(\bar{h}(M), N^{\prime}\right) \in \mathscr{B}^{\prime}$ implies, for instance, $\bar{h}(M)=w^{\prime}\left(a_{1}^{\prime} a_{2}^{\prime}, b^{\prime}\right), N^{\prime}$ $=w^{\prime}\left(a_{1}^{\prime}, b^{\prime}\right) w^{\prime}\left(a_{2}^{\prime}, b^{\prime}\right)$ for some $a_{1}^{\prime}, a_{2}^{\prime} \in A^{\prime}, b^{\prime} \in B$. If $M=w(a, b)$, then $a_{1}^{\prime} a_{2}^{\prime}=f(a)$, $b^{\prime}=g(b)$. Since $f$ is consistent, $a_{1}^{\prime}=f\left(a_{1}\right), a_{2}^{\prime}=f\left(a_{2}\right)$ for some $a_{1}, a_{2} \in A$; therefore $N^{\prime}=\bar{h}\left(w\left(a_{1}, b\right) w\left(a_{2}, b\right)\right) \in \operatorname{Im} \bar{h}$. The other case is treated similarly, using the consistency of $g$. In a similar manner, one shows that $\left(M^{\prime}, \bar{h}(N)\right) \in \mathscr{B}^{\prime}$ implies $M^{\prime} \in \operatorname{Im} h$.

From this follows immediately that $(\bar{h}(M), \bar{h}(N)) \in \overline{\mathscr{B}^{\prime}}$ implies $(M, N) \in \overline{\mathscr{B}}$ and that $\left(\bar{h}(M), N^{\prime}\right) \in \overline{\mathscr{B}}^{\prime}$ implies $N^{\prime} \in \operatorname{Im} \bar{h}$.

If now $(\bar{h}(M), \bar{h}(N)) \in \mathscr{T}^{\prime}$, then $\bar{h}(M)=U^{\prime} P^{\prime} V^{\prime}, \bar{h}(N)=U^{\prime} Q^{\prime} V^{\prime}$, where $U^{\prime}, V^{\prime}$ $\in F^{\prime 1}$ and $\left(P^{\prime}, Q^{\prime}\right) \in \overline{\mathscr{B}} \bar{B}^{\prime}$, and we can find $U, V \in F^{1}, P, Q \in F$ such that $\bar{h}(U)=U^{\prime}$, $\bar{h}(V)=V^{\prime}, \bar{h}(P)=P^{\prime}, \bar{h}(Q)=Q^{\prime}, M=U P V, N=U Q V$. Since $(\bar{h}(P), \bar{h}(Q)) \in \overline{\mathscr{B}^{\prime}}$, we have $(P, Q) \in \overline{\mathscr{B}}$ and therefore $(M, N) \in \mathscr{T}$.

Furthermore if $\left(\bar{h}(M), N^{\prime}\right) \in \mathscr{T}^{\prime}$, then $\bar{h}(M)=U^{\prime} P^{\prime} V^{\prime}, N^{\prime}=U^{\prime} Q^{\prime} V^{\prime}$, where $U^{\prime}, V^{\prime} \in F^{\prime 1}$ and $\left(P^{\prime}, Q^{\prime}\right) \in \overline{\mathscr{B}^{\prime}}$. Since $\bar{h}$ is consistent, $U^{\prime}, P^{\prime}, V^{\prime} \in \operatorname{Im} \bar{h}$; since $\left(P^{\prime}, Q^{\prime}\right) \in \overline{\mathscr{B}}^{\prime}$, we have also $Q^{\prime} \in \operatorname{Im} \bar{h}$ and $N^{\prime}=U^{\prime} Q^{\prime} V^{\prime} \in \operatorname{Im} \bar{h}$.

If finally $(\bar{h}(M), \bar{h}(N)) \in \mathscr{C}^{\prime}$, then we can find $P_{0}^{\prime}, P_{1}^{\prime}, \ldots, P_{n}^{\prime} \in F^{\prime}$ such that $P_{0}^{\prime}=\bar{h}(M),\left(P_{i}^{\prime}, P_{i+1}^{\prime}\right) \in \mathscr{T}^{\prime}$ for all possible $i$ and $P_{n}^{\prime}=\bar{h}(N)$. Since $\left(P_{0}^{\prime}, P_{1}^{\prime}\right) \in \mathscr{T}^{\prime}$ and $P_{0}^{\prime}=\bar{h}(M)$, we have $P_{1}^{\prime} \in \operatorname{Im} \bar{h}$, say $P_{1}^{\prime}=\bar{h}\left(P_{1}\right)$; and $\left(\bar{h}(M), \bar{h}\left(P_{1}\right)\right) \in \mathscr{T}^{\prime}$ implies $\left(M, P_{1}\right) \in \mathscr{T}$. Inductively, we obtain $P_{i}^{\prime}=\bar{h}\left(P_{i}\right)$ for some $P_{i} \in F$ and $\left(P_{i}, P_{i+1}\right) \in \mathscr{T}$, for all possible $i$. Eventually $\bar{h}\left(P_{n}\right)=\bar{h}(N)$, so that $P_{n}=N$. Therefore $(M, N) \in \mathscr{C}$.

From this we conclude that $f \otimes g$ is one-to-one.

Furthermore, proceeding as above, we see that $\left(\bar{h}(M), N^{\prime}\right) \in \mathscr{C}^{\prime}$ implies $N^{\prime} \in \operatorname{Im} \bar{h}$. Therefore $k^{\prime}\left(M^{\prime}\right) k^{\prime}\left(N^{\prime}\right)=(f \otimes g)(k(P))$ implies successively: $\left(M^{\prime} N^{\prime}, \bar{h}(P)\right) \in \mathscr{C}^{\prime}$; $M^{\prime} N^{\prime}=\bar{h}(Q)$ for some $Q \in F ; M^{\prime}=\bar{h}(M), N^{\prime}=\bar{h}(N)$ for some $M, N \in F$; and finally $k^{\prime}\left(M^{\prime}\right)=k^{\prime}(\bar{h}(M))=(f \otimes g)(k(M)) \in \operatorname{Im} f \otimes g, k^{\prime}\left(N^{\prime}\right) \in \operatorname{Im} f \otimes g$, therefore $f \otimes g$ is consistent (since $k, k^{\prime}$ are onto) which completes the proof of the theorem.

Using this result, we can extend Theorem 3.4 to the case when $f$ and $g$ are consistent.

THEOREM 4.3. If $f$ and $g$ are consistent, then $\operatorname{ker} f \otimes g=\operatorname{ker} f \otimes \operatorname{ker} g$; furthermore $\operatorname{Im} f \otimes g \cong \operatorname{Im} f \otimes \operatorname{Im} g$, and $f \otimes g$ is consistent.

Proof. Let $i(j)$ be the inclusion mapping of $\operatorname{Im} f(\operatorname{Im} g)$ into $A^{\prime}\left(B^{\prime}\right)$, and $q(r)$ be the canonical homomorphism of $A(B)$ onto $\operatorname{Im} f(\operatorname{Im} g)$, so that $f=i \circ q(g=j \circ r)$. 
Since $\operatorname{Im} i=\operatorname{Im} f, \operatorname{Im} j=\operatorname{Im} g, i$ and $j$ are consistent. Then $f \otimes g=(i \otimes j) \circ(q \otimes r)$; since $i \otimes j$ is one-to-one by 4.1: $\operatorname{ker} f \otimes g=\operatorname{ker} q \otimes r=\operatorname{ker} q \otimes \operatorname{ker} r=\operatorname{ker} f$ $\otimes$ ker $g$ by 3.4. Furthermore, $\operatorname{Im} f \otimes g \cong \operatorname{Im} q \otimes r=\operatorname{Im} f \otimes \operatorname{Im} g$. Finally, $i \otimes j$ is consistent by Theorem 4.1, so that $f \otimes g$ is also consistent.

5. "Right exactness" and adjoint associativity. In this section, we show that the tensor product of semigroups keeps, to a certain extent, the right exactness and adjoint associativity properties of the tensor product of modules.

While exactness is self-dual in an abelian category, its natural generalization to a nonabelian category like $\mathscr{S}$ is not (see [3]). Therefore we shall consider two kinds of "exact" sequences. We say that a sequence $A^{\prime} \stackrel{f}{\rightarrow} A \stackrel{f^{\prime}}{\rightarrow} A$ " is exact if $\operatorname{Im} f$ is a class of $\operatorname{ker} f^{\prime}$, and coexact (cf. [3]) if $\operatorname{ker} f^{\prime}$ is the smallest congruence $\mathscr{K}$ on $A$ such that $\operatorname{Im} f$ is contained in only one class of $\mathscr{K}$; if the sequence above is either exact or coexact, then it is null, i.e. $\operatorname{Im}\left(f^{\prime} \circ f\right)$ consists only of one (idempotent) element of $A^{\prime \prime}$. For a null sequence, exactness, coexactness do not imply each other; if, however, the sequence $A^{\prime} \stackrel{f}{\rightarrow} A \stackrel{f^{\prime}}{\rightarrow} A^{\prime \prime}$ is coexact and if $\operatorname{Im} f$ is an ideal of $A$, then the sequence is exact (and $A$ is an ideal extension of $\operatorname{Im} f$ by $\operatorname{Im} f^{\prime}$ ).

Another difficulty is that, if $A^{\prime} \stackrel{f}{\rightarrow} A \stackrel{f^{\prime}}{\rightarrow} A^{\prime \prime}$ is null, then $A^{\prime} \otimes X \rightarrow A \otimes X \rightarrow A^{\prime \prime}$ $\otimes X$ need not be null. We shall see in fact that there is no trouble if $X$ is $s$-indecomposable (i.e. if the largest idempotent homomorphic image of $X$ is trivial, cf. [7]).

THEOREM 5.1. If $A^{\prime} \stackrel{f}{\rightarrow} A \stackrel{f^{\prime}}{\rightarrow} A^{\prime \prime}$ is coexact and if $X$ is s-indecomposable and $f^{\prime}$ consistent (for instance, onto), then

$$
A^{\prime} \otimes X \stackrel{f \otimes \mathrm{id}_{x}}{\longrightarrow} A \otimes X \stackrel{f^{\prime} \otimes \mathrm{id}_{x}}{\longrightarrow} A^{\prime \prime} \otimes X
$$

is coexact.

Proof. Set $f \otimes \mathrm{id}_{X}=\bar{f}, f^{\prime} \otimes \mathrm{id}_{X}=\bar{f}$. First we prove that $A^{\prime} \otimes X \stackrel{f}{\rightarrow} A \otimes X \stackrel{f^{\prime}}{\rightarrow} A^{\prime \prime}$ $\otimes X$ is null. Define a homomorphism $p$ of $X$ into $A^{\prime \prime} \otimes X$ by: $p(x)=e^{\prime \prime} \otimes x$, where $\left\{e^{\prime \prime}\right\}=\operatorname{Im}\left(f^{\prime} \circ f\right)$. Since $e^{\prime \prime}$ is idempotent, every element of $\operatorname{Im} p$ is idempotent and, by the condition on $X, \operatorname{Im} p$ is trivial, say $\operatorname{Im} p=\left\{\bar{e}^{\prime \prime}\right\}$, where $\bar{e}^{\prime \prime} \in A^{\prime \prime} \otimes X$ is necessarily idempotent. Now

$$
\bar{f}^{\prime}(\bar{f}(a \otimes x))=f^{\prime}(f(a)) \otimes x=e^{\prime \prime} \otimes x=\bar{e}^{\prime \prime}
$$

for all $a \in A, x \in X$. Since $A \otimes X$ is generated by all elements of the form $a \otimes x$, it follows that $\operatorname{Im} \bar{f}^{\prime} \circ \bar{f}=\left\{e^{\prime \prime}\right\}$. This shows that our sequence is null. In other words, $\operatorname{Im} \bar{f}$ is contained in only one class of $\operatorname{ker} \bar{f}^{\prime}$.

On the other hand, $\operatorname{ker} f^{\prime}$ is the smallest congruence on $A$ containing all pairs $\left(f\left(a_{1}\right), f\left(a_{2}\right)\right)\left(a_{1}, a_{2} \in A^{\prime}\right)$, by coexactness: so that, by Theorem 4.3 and Lemma 3.6, $\operatorname{ker} \bar{f}^{\prime}$ is the smallest congruence on $A \otimes X$ containing all pairs

$$
\left(f\left(a_{1}\right) \otimes x, f\left(a_{2}\right) \otimes x\right),
$$


where $a_{1}, a_{2} \in A, x \in X$. But any congruence with the property that $\operatorname{Im} \bar{f}$ is contained in only one class contains all these pairs, and so must contain $\operatorname{ker} \bar{f}^{\prime}$. This means that $A^{\prime} \otimes X \stackrel{f}{\rightarrow} A \otimes X \stackrel{f^{\prime}}{\rightarrow} A^{\prime \prime} \otimes X$ is coexact.

COROLlaRY 5.2. If $A^{\prime} \stackrel{f}{\rightarrow} A \stackrel{f^{\prime}}{\rightarrow} A^{\prime \prime}$ is coexact and if $f^{\prime}$ is consistent, then $N\left(A^{\prime}\right)$ $\rightarrow N(A) \rightarrow N\left(A^{\prime \prime}\right)$ is coexact.

COROllaRy 5.3. If $A^{\prime} \stackrel{f}{\rightarrow} A \stackrel{f^{\prime}}{\rightarrow} A^{\prime \prime}$ is coexact and if $f^{\prime}$ is consistent, then $E\left(A^{\prime}\right)$ $\rightarrow E(A) \rightarrow E\left(A^{\prime \prime}\right)$ is coexact.

Proof. The corollaries follow from Propositions 1.2, 1.1, since any infinite cyclic semigroup, any one-element semigroup, are $s$-indecomposable.

Whether the tensor product by an $s$-indecomposable semigroup preserves exact sequences $A^{\prime} \stackrel{f}{\rightarrow} A \stackrel{f^{\prime}}{\rightarrow} A^{\prime \prime}$ (with $f^{\prime}$ onto) is a question we could not solve. Yet we have the following result.

THEOREM 5.4. Let $A^{\prime} \stackrel{f}{\rightarrow} A \stackrel{f^{\prime}}{\rightarrow} A^{\prime \prime}$ be an exact and coexact sequence, where $f$ and $f^{\prime}$ are consistent. Let $X$ be an s-indecomposable semigroup. Then the sequence

$$
A^{\prime} \otimes X \stackrel{f \otimes \mathrm{id}_{x}}{\longrightarrow} A \otimes X \stackrel{f^{\prime} \otimes \mathrm{id}_{x}}{\longrightarrow} A^{\prime \prime} \otimes X
$$

is exact and coexact. Furthermore $f \otimes \mathrm{id}_{X}, f^{\prime} \otimes \mathrm{id}_{X}$ are consistent and if $f$ is one-toone, so is $f \otimes \mathrm{id}_{x}$.

Proof. Observe that a nonempty consistent subsemigroup $K$ of a semigroup $S$ is a class of some congruence on $S$ (since $S-K$ is either empty or a prime ideal). Since by Theorem $4.3 f \otimes \mathrm{id}_{X}$ is consistent, $\operatorname{Im} f \otimes \mathrm{id}_{X}$ is a class of some congruence on $A \otimes X$ and by coexactness (by Theorem 5.1) must be a class of ker $f^{\prime}$ $\otimes \mathrm{id}_{\mathrm{X}}$. The other conclusions of the theorem follow from Theorems 4.1, 4.3.

The reader will be able to state the corresponding corollaries concerning $N(A)$, $E(A)$.

Finally we turn to the adjoint associativity. A first difficulty is that the set $\operatorname{Mor}_{\mathscr{S}}(A, B)$ of all semigroup homomorphisms of $A$ into $B$ may be empty and in general will not inherit more than a structure of a partial groupoid. By a partial groupoid we mean, following [1], a set $P$ together with a partial binary operation. A homomorphism of a partial groupoid $P$ into a partial groupoid $Q$ is a mapping $f$ of $P$ into $Q$ such that $f(x) \cdot f(y)$ is defined in $Q$ and equal to $f(x \cdot y)$ whenever $x \cdot y$ is defined in $P$. Partial groupoids and their homomorphisms obviously form a category which shall be denoted by $\mathscr{P}$. The category $\mathscr{S}$ of semigroups is a full subcategory of $\mathscr{P}$. Finally the set $\operatorname{Mor}_{\mathscr{P}}(P, Q)$ of all partial groupoid homomorphisms of $P$ into $Q$ inherits a structure of partial groupoid in the following fashion: If $f, g, h \in \operatorname{Mor}_{\mathscr{P}}(P, Q)$ we say that $f \cdot g=h$ if and only if $f(x) \cdot g(x)$ is defined in $Q$ and equal to $h(x)$ for all $x \in P$. If in particular $P$ and $Q$ are semigroups, $\operatorname{Mor}_{\mathscr{S}}(P, Q)$ becomes a partial groupoid, which can always be embedded into a semigroup but need not be one. The following result is of interest. First, call a semigroup $S$ 
subcommutative if and only if the identity $x y z t=x z y t$ holds in $S$ (various other names for these semigroups can be found in the literature). Clearly, any commutative semigroup is subcommutative, and any subcommutative semigroup is normal.

Proposition 5.5. Let $B$ be a semigroup. Then $\operatorname{Mor}_{\mathscr{S}}(A, B)$ is a semigroup for any semigroup $A$ if and only if $B$ is subcommutative.

Proof. Clearly $\operatorname{Mor}_{\mathscr{S}}(A, B)$ is a semigroup if and only if $f \cdot g$ is defined for all $f, g \in \operatorname{Mor}_{\mathscr{S}}(A, B)$. If $B$ is subcommutative, then the mapping $x \rightarrow f(x) g(x)$ of $A$ into $B$ is a homomorphism for any $f, g \in \operatorname{Mor}_{\mathscr{S}}(A, B)$, so that $f \cdot g$ is always defined. Suppose conversely that $\operatorname{Mor}_{\mathscr{S}}(A, B)$ is a semigroup for any semigroup $A$ and take $x, y, z, t \in B$. Let $A$ be the free semigroup on two generators $a^{\prime}, a^{\prime \prime}$, and $f(g)$ be the homomorphism of $A$ into $B$ such that $f\left(a^{\prime}\right)=x, f\left(a^{\prime \prime}\right)=z\left(g\left(a^{\prime}\right)=y, g\left(a^{\prime \prime}\right)=t\right)$. Since $f \cdot g$ is defined,

$$
\begin{aligned}
x y z t & =f\left(a^{\prime}\right) g\left(a^{\prime}\right) f\left(a^{\prime \prime}\right) g\left(a^{\prime \prime}\right)=(f \cdot g)\left(a^{\prime}\right)(f \cdot g)\left(a^{\prime \prime}\right)=(f \cdot g)\left(a^{\prime} a^{\prime \prime}\right) \\
& =f\left(a^{\prime}\right) f\left(a^{\prime \prime}\right) g\left(a^{\prime}\right) g\left(a^{\prime \prime}\right)=x z y t .
\end{aligned}
$$

Therefore $B$ is subcommutative, which completes the proof.

TheOREM 5.6. Let $A, B, C$ be semigroups. There exists a canonical one-to-one homomorphism of $\operatorname{Mor}_{\mathscr{S}}(A \otimes B, C)$ onto $\operatorname{Mor}_{\mathscr{P}}\left(A, \operatorname{Mor}_{\mathscr{S}}(B, C)\right)$, which is natural in $A, B, C$ and becomes a semigroup isomorphism if $C$ is subcommutative.

Proof. Define a mapping $\varphi$ by: $((\varphi(u))(a))(b)=u(a \otimes b)$ for all $a \in A, b \in B$, $u \in \operatorname{Mor}_{\mathscr{S}}(A \otimes B, C)$. Then, for any $a, a^{\prime}, a^{\prime \prime} \in A, \quad b, b^{\prime}, b^{\prime \prime} \in B, u, u^{\prime}, u^{\prime \prime} \in$ $\operatorname{Mor}_{\mathscr{S}}(A \otimes B, C)$ :

$$
\begin{aligned}
((\varphi(u))(a))\left(b^{\prime} b^{\prime \prime}\right) & =u\left(a \otimes b^{\prime} b^{\prime \prime}\right)=u\left(a \otimes b^{\prime}\right) u\left(a \otimes b^{\prime \prime}\right) \\
& =((\varphi(u))(a))\left(b^{\prime}\right)((\varphi(u))(a))\left(b^{\prime \prime}\right),
\end{aligned}
$$

whence $(\varphi(u))(a) \in \operatorname{Mor}_{\mathscr{S}}(B, C)$;

$$
\begin{aligned}
\left((\varphi(u))\left(a^{\prime} a^{\prime \prime}\right)\right)(b) & =u\left(a^{\prime} a^{\prime \prime} \otimes b\right)=u\left(a^{\prime} \otimes b\right) u\left(a^{\prime \prime} \otimes b\right) \\
& =\left((\varphi(u))\left(a^{\prime}\right)\right)(b)\left((\varphi(u))\left(a^{\prime \prime}\right)\right)(b) ;
\end{aligned}
$$

whence $\varphi(u) \in \operatorname{Mor}_{\mathscr{P}}\left(A, \operatorname{Mor}_{\mathscr{S}}(B, C)\right)$;

$$
\begin{aligned}
\left(\left(\varphi\left(u^{\prime} \cdot u^{\prime \prime}\right)\right)(a)\right)(b) & =\left(u^{\prime} \cdot u^{\prime \prime}\right)(a \otimes b)=u^{\prime}(a \otimes b) u^{\prime \prime}(a \otimes b) \\
& =\left(\left(\varphi\left(u^{\prime}\right)\right)(a)\right)(b)\left(\left(\varphi\left(u^{\prime \prime}\right)\right)\left(a^{\prime \prime}\right)\right)(b)
\end{aligned}
$$

whenever $u^{\prime} \cdot u^{\prime \prime}$ is defined, whence

$$
\begin{aligned}
\left(\varphi\left(u^{\prime} \cdot u^{\prime \prime}\right)\right)(a) & =\left(\varphi\left(u^{\prime}\right)\right)(a) \cdot\left(\varphi\left(u^{\prime \prime}\right)\right)(a), \varphi\left(u^{\prime} \cdot u^{\prime \prime}\right) \\
& =\varphi\left(u^{\prime}\right) \cdot \varphi\left(u^{\prime \prime}\right)
\end{aligned}
$$

and $\varphi$ is a homomorphism.

Let also $\sigma$ be a homomorphism of $A$ into $\operatorname{Mor}_{\mathscr{S}}(B, C)$. For all $a \in A, b \in B$, define $s(a, b)=(\sigma(a))(b)$. Then $s$ is bilinear. Therefore one can find

$$
u \in \operatorname{Mor}_{\mathscr{S}}(A \otimes B, C)
$$


such that $s(a, b)=u(a \otimes b)$, i.e. $(\sigma(a))(b)=((\varphi(u))(a))(b)$ for all $a, b$, i.e. $\sigma=\varphi(u)$. Therefore $\varphi$ is onto; since furthermore $u$ is unique above, $\varphi$ is also one-to-one.

Finally, if $C$ is subcommutative, then $\operatorname{Mor}_{\mathscr{S}}(A \otimes B, C)$ and $\operatorname{Mor} \mathscr{S}(B, C)$ are semigroups; furthermore it is immediate that $\operatorname{Mor}_{\mathscr{S}}(B, C)$ is also subcommutative, so that $\operatorname{Mor}_{\mathscr{P}}\left(A, \operatorname{Mor}_{\mathscr{S}}(B, C)\right)$ is a semigroup too. Therefore $\varphi$ is then a semigroup isomorphism. Naturality is left to the reader.

It should be noted that if $C$ is not subcommutative, then $\varphi$ need not be an isomorphism. For example, take $A$ and $B$ as in Example 2.3 and $C=A \otimes B$. Then $\operatorname{id}_{C} \in \operatorname{Mor}_{\mathscr{S}}(A \otimes B, A \otimes B) ; \mathrm{id}_{C} \cdot \mathrm{id}_{C}$ is not defined in that partial semigroup since

$$
\left(\mathrm{id}_{C} \cdot \mathrm{id}_{C}\right)(e)\left(\mathrm{id}_{C} \cdot \mathrm{id}_{C}\right)(f)=e e f f=e f \neq(e f)^{2}=.\left(\mathrm{id}_{C} \cdot \mathrm{id}_{C}\right)(e f)
$$

However $\sigma=\varphi\left(\mathrm{id}_{C}\right)$ is defined by $(\sigma(x))(y)=x \otimes y$ for all $x \in A, y \in B$; that is, $(\sigma(0))(0)=0,(\sigma(1))(1)=1,(\sigma(0))(1)=e,(\sigma(1))(0)=f$. Hence $\sigma \cdot \sigma$ is defined in

$$
\operatorname{Mor}_{\mathscr{P}}\left(A, \operatorname{Mor}_{\mathscr{S}}(B, A \otimes B)\right)
$$

and equal to $\sigma$, since $0,1, e, f$ are idempotents. Hence $\varphi$ is not in this case an isomorphism.

On the other hand, $\varphi$ may be an isomorphism even though $C$ is not subcommutative. Let $C$ be any idempotent semigroup, for instance the semigroup given by the table

\begin{tabular}{l|llll} 
& $a$ & $b$ & $c$ & $d$ \\
\hline$a$ & $a$ & $a$ & $a$ & $a$ \\
$b$ & $b$ & $b$ & $b$ & $b$ \\
$c$ & $a$ & $b$ & $c$ & $d$ \\
$d$ & $a$ & $b$ & $c$ & $d$
\end{tabular}

(which is not subcommutative since $c a b d=a, c b a d=b$ ). Let $A, B$ be any one element semigroups. Then $\operatorname{Mor}_{\mathscr{S}}(B, C) \cong C$; similarly, $\operatorname{Mor}_{\mathscr{P}}\left(A, \operatorname{Mor}_{\mathscr{S}}(B, C)\right)$ and $\operatorname{Mor}_{\mathscr{S}}(A \otimes B, C)$ are isomorphic to $C$, since $A$ and $A \otimes B$ have only one element. In this case, $\varphi$ is the natural isomorphism

$$
\operatorname{Mor}_{\mathscr{S}}(A \otimes B, C) \cong C \cong \operatorname{Mor}_{\mathscr{P}}\left(A, \operatorname{Mor}_{\mathscr{S}}(B, C)\right) \text {. }
$$

6. Tensor product in $\mathscr{S}_{0}$. By $\mathscr{S}_{0}$ we understand the category whose objects are all semigroups with zero (all the zeros shall be denoted by 0 ) and whose morphisms are all semigroup homomorphisms $f$ such that $f(0)=0$.

If $\left(A_{\imath}\right)_{i \in I}$ is any family of semigroups with zero, a mapping $s$ of $\prod_{i \in I} A_{i}$ into some semigroup with zero $C$ is zero-I-linear if for every $i_{0} \in I$, the mapping $a_{i_{0}} \leadsto s\left(\left(a_{i}\right)_{i \in I}\right)$ is a homomorphism of $\mathscr{S}_{0}$ of $A_{i_{0}}$ into $C$ whenever all $a_{i}$ are fixed except for $a_{i_{0}}$. In other words, $s$ is zero-I-linear if and only if it is $I$-linear and satisfies $s\left(\left(a_{\imath}\right)_{i \in I}\right)=0$ whenever one of the $a_{i}$ is 0 . Expressions like zerobilinear, zerotrilinear, shall have 
the obvious meaning when $I$ has few elements. We define a zero tensor product of the family just as we defined the tensor product, but replacing $I$-linear mappings by zero-I-linear mappings and homomorphisms by homomorphisms of $\mathscr{S}_{0}$. We keep the same notation, with $\otimes^{0}$ instead of $\otimes$.

Observe that, if each $A_{i}$ is a semigroup with zero, $\bigotimes_{i \in I} A_{i}$ will also be a semigroup with a zero (namely, $\bigotimes_{i \in I}^{0}$ ) but need not be a zero tensor product of $\left(A_{i}\right)_{i \in I}$; a counterexample is furnished by Example 2.3 in which $0 \otimes 1 \neq 0$.

It is easy to show the existence of $\bigotimes_{i \in I}^{0} A_{i}$ for any family $\left(A_{i}\right)_{i \in I}$ of semigroups with zero, using the same method as in Theorem 2.1 and an analogous construction. The following result gives a quicker construction and establishes a relationship between $\otimes^{\circ}$ and $\otimes$.

THEOREM 6.1. Let $\left(A_{i}\right)_{i \in I}$ be any family of semigroups with zero. Let $Z$ be the ideal of $\bigotimes_{i \in I} A_{i}$ generated by all elements $\bigotimes_{i \in I} a_{i}$ such that $a_{i}=0$ for some $i$. Then $\bigotimes_{i \in I} A_{i} / Z$ is a zero tensor product of $\left(A_{i}\right)_{i \in I}$.

Proof. Let $p$ be the canonical homomorphism of $\bigotimes_{i \in I} A_{i}$ onto $\bigotimes_{i \in I} A_{i} / Z$. Then $\bigotimes_{i \in I} A_{i} / Z$ is a semigroup with zero and $t:\left(a_{i}\right)_{i \in I} \leadsto p\left(\bigotimes_{i \in I} a_{i}\right)$ a zero-I-linear mapping. Let $s$ be any zero-I-linear mapping of $\prod_{i \in I} A_{i}$ into some semigroup with zero $C$. Since $s$ is in particular $I$-linear, there is a unique homomorphism $u$ of $\bigotimes_{i \in I} A_{i}$ into $C$ such that $s\left(\left(a_{i}\right)_{i \in I}\right)=u\left(\bigotimes_{i \in I} a_{i}\right)$ identically. Since $s$ is zero-I-linear,

$$
u\left(\otimes_{i \in I} a_{i}\right)=0
$$

whenever $a_{i}=0$ for some $i$; hence $u$ is null on $Z$ and there exists a unique homomorphism $v$ of $\bigotimes_{i \in I} A_{i} / Z$ into $C$ such that $u=v \circ p$. Then $v$ is also such that $v \circ t=s$. Since $\bigotimes_{i \in I} A_{i} / Z$ is generated by $\operatorname{Im} t$, such $v$ is unique, which completes the proof.

For the next result, we switch for simplicity to the case when $I$ has two elements. For any semigroup $S$, denote by $S^{0}$ any semigroup resulting from the adjunction of a formal zero to $S$.

TheOREM 6.2. If $A, B$ are any semigroups, $A^{0} \otimes^{0} B^{0} \cong(A \otimes B)^{0}$.

Proof. Let $f(g)$ be the canonical inclusion homomorphism of $A(B)$ into $A^{0}\left(B^{0}\right)$. Since $f$ and $g$ are one-to-one and consistent, the homomorphism $f \otimes g$ of $A \otimes B$ into $A^{0} \otimes B^{0}$ is consistent by Theorem 4.1. In particular $Y=A^{0} \otimes B^{0}-\operatorname{Im} f \otimes g$ is either empty or an ideal of $A^{0} \otimes B^{0}$. We are going to prove that $Y$ coincides with the ideal $Z$ of $A^{0} \otimes B^{0}$.generated by all elements of the form $0 \otimes b^{\prime}, a^{\prime} \otimes 0$ $\left(a^{\prime} \in A, b^{\prime} \in B\right)$.

We keep the notation of 3.1, the primes indicating now that we deal with $A^{0} \otimes B^{0}$. First it follows from the proof of Theorem 4.1 that $\left(\bar{h}(M), N^{\prime}\right) \in \mathscr{C}^{\prime}$ implies $N^{\prime} \in \operatorname{Im} \bar{h}$. Next it is clear that $M^{\prime}=w^{\prime}\left(a_{1}^{\prime}, b_{1}^{\prime}\right) \cdots w^{\prime}\left(a_{n}^{\prime}, b_{n}^{\prime}\right)$ is in $\operatorname{Im} \bar{h}$ if and only if $a_{i}^{\prime} \neq 0, b_{i}^{\prime} \neq 0$ for all $i$. From this we deduce that $Z \subseteq Y$. If $t^{\prime} \in Z$, then it can be written under the form $t^{\prime}=\left(a_{1}^{\prime} \otimes b_{1}^{\prime}\right) \cdots\left(a_{n}^{\prime} \otimes b_{n}^{\prime}\right)$, where $a_{i}^{\prime} \in A, b_{i}^{\prime} \in B$ for all $i$, 
and $a_{i}^{\prime}=0$ or $b_{i}^{\prime}=0$ for some $i$. Setting $N^{\prime}=w^{\prime}\left(a_{1}^{\prime}, b_{1}^{\prime}\right) \cdots w^{\prime}\left(a_{n}^{\prime}, b_{n}^{\prime}\right)$, so that $t^{\prime}$ $=k^{\prime}\left(N^{\prime}\right)$, we conclude that $N^{\prime} \notin \operatorname{Im} \bar{h}$. If now $t^{\prime} \notin Y$, then $t^{\prime} \in \operatorname{Im} f \otimes g$ and, for some $M \in F, t^{\prime}$ would be equal to $(f \otimes g)(k(M))=k^{\prime}(\bar{h}(M))$; then $\left(\bar{h}(M), N^{\prime}\right) \in \mathscr{C}^{\prime}$ and $N^{\prime} \in \operatorname{Im} \bar{h}$, which is impossible.

On the other hand, if $t^{\prime} \in Y$, then $t^{\prime} \notin \operatorname{Im} f \otimes g$. If $t^{\prime}$ is written under the form $k^{\prime}\left(M^{\prime}\right)$ for some $M^{\prime}=w^{\prime}\left(a_{1}^{\prime}, b_{1}^{\prime}\right) \cdots w^{\prime}\left(a_{n}^{\prime}, b_{n}^{\prime}\right)$, then $M^{\prime} \notin \operatorname{Im} \bar{h}$ and therefore $a_{i}=0$ or $b_{i}=0$ for some $i$. Hence $t^{\prime}=\left(a_{1}^{\prime} \otimes b_{1}^{\prime}\right) \cdots\left(a_{n}^{\prime} \otimes b_{n}^{\prime}\right) \in Z$.

Therefore $Y=Z$. Since $\operatorname{Im} f \otimes g$ is a subsemigroup of $A^{0} \otimes B^{0}$, we have

$$
A^{0} \otimes B^{0} / Z=A^{0} \otimes B^{0} / Y \cong(\operatorname{Im} f \otimes g)^{0} .
$$

Since $f \otimes g$ is one-to-one by Theorem 4.1, we conclude that

$$
(A \otimes B)^{0} \cong(\operatorname{Im} f \otimes g)^{0} \cong A^{0} \otimes B^{0} / Z \cong A^{0} \otimes^{0} B^{0},
$$

in view of Theorem 6.1. This completes the proof.

It is immediate to verify that, except for 1.1 and 5.3 , all properties of $\S \S 1,3,5$ extend to $\otimes^{0}$ (replacing $B$ by $B^{0}$ in $1.2,1.3$ ). We do not mention $\S 4$, since a consistent homomorphism of $\mathscr{S}_{0}$ is necessarily onto. Yet the main reason we introduced $\otimes^{0}$ is that 5.1 improves with $\otimes^{0}$. Namely, we can now lift all restriction on $X$ in 5.1 to obtain:

THEOREM 6.3. Let $A^{\prime} \stackrel{f}{\rightarrow} A \stackrel{f^{\prime}}{\rightarrow} A^{\prime \prime}$ be a coexact sequence (of $\mathscr{S}_{0}$ ), where $f^{\prime}$ is onto, and let $X$ be any semigroup with zero. Then the following sequence:

$$
A^{\prime} \otimes^{0} X \stackrel{f \otimes^{0} \mathrm{id}_{x}}{\longrightarrow} A \otimes^{0} X \stackrel{f^{\prime} \otimes^{0} \mathrm{id}_{x}}{\longrightarrow} A^{\prime \prime} \otimes^{0} X
$$

is coexact.

Proof. The reason is that $X \otimes^{0}\{0\}=\{0\}$ by the definition of a zerobilinear mapping. With the same notation as in 5.1 (except for $\otimes^{0}$ ), we have $e^{\prime \prime}=0$, and $e^{\prime \prime} \otimes^{0}$ $x=0$ for all $x \in X$ (even if $X$ is not $s$-indecomposable). Using the same reasoning as in the proof of 5.1 , one concludes that the sequence $A^{\prime} \otimes^{0} X \rightarrow A \otimes^{0} X \rightarrow A^{\prime \prime}$ $\otimes^{0} X$ is null, then coexact.

\section{REFERENCES}

1. A. H. Clifford and G. B. Preston, The algebraic theory of semigroups, Vol. 1, Math. Surveys, No. 7, Amer. Math. Soc., Providence, R. I., 1962, reprint 1964.

2. P. Dubreil, Contribution à la théorie des demi-groupes, Mém. Acad. Sci. Inst. France (2) 63 (1941), $52 \mathrm{pp}$.

3. P. A. Grillet, Quelques propriétés des catégories nonabéliennes, C. R. Acad. Sci. Paris Sér. A 266 (1968), 550-552.

4. - La suite exacte d'homologie dans une catégorie non-abélienne, C. R. Acad. Sci. Paris Sér. A 266 (1968), 604-606.

5. N. Kimura, Identity $(x y)^{n}=x^{n} y^{n}$ on semigroups, (to appear).

6. S. MacLane, Homology, Springer-Verlag, Berlin, 1963.

7. T. Tamura and N. Kimura, Existence of a greatest decomposition of a semigroup, Kōdai Math. Sem. Rep. 7 (1955), 83-84.

Kansas State University, Manhattan, Kansas 